\title{
Superelastic Cyclic Properties of Cu-Al-Mn and Ni-Ti Shape Memory Alloys for Seismic Mitigation
}

\author{
Burak Duran ${ }^{1,2}$, Kadri Can Atlı ${ }^{3}$, Özgür Avşar ${ }^{4}$ \\ ${ }^{1}$ Department of Civil, Structural and Environmental Engineering, University at Buffalo \\ Buffalo, NY, 14260, United States of America \\ burakdur@buffalo.edu \\ ${ }^{2}$ Formerly; Department of Civil Engineering, Dokuz Eylul University \\ Tinaztepe Campus, 35190, Buca, Izmir, Turkey \\ ${ }^{3}$ Department of Materials Science and Engineering, Texas A\&M University \\ College Station, TX, 77840, United States of America \\ catli@tamu.edu \\ ${ }^{4}$ Department of Civil Engineering, Eskisehir Technical University \\ 2 Eylul Campus, 26555, Eskisehir, Turkey \\ ozguravsar@eskisehir.edu.tr
}

\begin{abstract}
Shape memory alloys (SMAs) have been getting much attention by many researchers in a variety of application areas due to their unique properties of superelasticity (SE) and shape memory effect (SME). They have the ability to recover large inelastic deformations upon heating (SME) and stress removal (SE). In recent years, structural engineers have been dealing with these smart materials to incorporate into civil engineering applications such as rebar in the reinforcement of concrete structures, repairing, retrofitting, base isolation system, dampers for vibrational control, etc. To overcome and mitigate the possible seismic risk of the structure under consideration, understanding the material characteristics of SMAs under various loading conditions is one of the critical steps. In this study, the mechanical properties of two popular SE SMAs, i.e. copper-aluminum-manganese (Cu-Al-Mn) and nickel-titanium (Ni-Ti), were investigated in detail. Moreover, the mechanical properties of the conventional rebar steel were also identified for comparison purposes. Room temperature monotonic and incremental cyclic tests were applied on dog-bone shaped Steel, $\mathrm{Cu}-\mathrm{Al}-\mathrm{Mn}$ and Ni-Ti tensile coupon specimens the obtain and compare their mechanical characteristics. The results showed that $\mathrm{Cu}-\mathrm{Al}-\mathrm{Mn}$ and Ni-Ti materials exhibited a significant re-centering ability upon unloading with negligible and comparable residual deformations whereas the Steel experienced higher permanent plastic deformations with almost 3\% recovery at the same amount of deformation. In addition, the decrease in the amount of dissipated energy for $\mathrm{Cu}-\mathrm{Al}-\mathrm{Mn}$ and $\mathrm{Ni}-\mathrm{Ti}$ for consecutive cyclic motion is much less than conventional steel. Test results were also evaluated in terms of cyclic performance of materials, residual strain, recovery capacity, dissipated energy and equivalent viscous damping. Experimental outcomes highlighted the potential usage of SMAs in seismic applications and supply basis information for continued research.
\end{abstract}

Keywords: Shape memory alloys; Material testing; Cyclic tensile behavior; Seismic recovery; Residual deformation.

\section{Introduction}

Shape memory alloys (SMAs) are a class of metallic materials with extraordinary properties due to its unique atomic bonding leading to remembering its original shape after deformation. Based on the intensive research, especially done in the last two decades, SMAs have found an application potential itself in many engineering disciplines. The reason for the growing popularity of SMAs is their capability of sustaining a large amount of inelastic deformations with negligible residual strains (superelasticity, SE) compared to conventional materials. From a civil engineering point of view, the advancements in superelastic alloys introduce new opportunities and innovative solutions to structural engineering problems with an extensive range of applications [1].

The most prominent features of the SMA materials are the high-energy dissipation capacity, hysteretic damping, excellent fatigue, large strain and re-centering abilities with negligible residual deformations [2]. These distinctive properties make them smart materials; therefore, structural engineers have been investigating the effect of using these materials in seismic applications. There are several studies showing the potential usage of SMAs in the field of structural engineering area; retrofitting, repairing, base isolation and in a form of several structural components such as bridge restrainer, seismic reinforcement, prestressing members, etc. [2,3]. Although the Ni-based SMAs are the most popular ones in the research area, much attention has been drawn to $\mathrm{Cu}$-based SMAs due to their advantages in the cost of raw materials, machining and 
threading process [4]. Thus, Cu-based SMAs can be considered as an alternative to Ni-based ones. In this sense, understanding the nature of SMAs and determining the mechanical properties of these materials properly is critically important before using them in real applications. Superelastic Ni-Ti SMAs in the bar form was also surveyed by [5] to probe their candidacy in seismic applications. This study concluded that superelastic Ni-Ti bars can be used for earthquake engineering applications, specifically in bracing elements in buildings and restraining elements in bridges.

Within the context of this study, monotonic and cyclic tension tests were carried out on the dog-bone shaped of the two different superelastic SMA coupon specimens, which are Nickel-Titanium (Ni-Ti) and Copper-Manganese-Aluminum (CuAl-Mn) in order to characterize mechanical and shape memory properties, and probe their prospective usage in civil engineering applications. Furthermore, the same tests were also conducted on conventional steel samples to make a comparison between the results. To explore the advantages and disadvantages of the materials in detail, some significant parameters like residual strain, recovery capacity, energy dissipation capacity and equivalent viscous damping were presented in this paper.

\section{Fundamental Concepts of Shape Memory Alloys}

The term "shape memory" comes from remembering the original shape after deformation. SMAs can sustain high postelastic deformations and recover their deformed shapes upon the removal of the applied loading (stress-induced martensitic transformation, SIM) or by means of heating effect (temperature-induced martensitic transformation,). The former can be achieved by only stress excitation through the superelasticity of the materials whereas the latter can be implemented by applying temperature excitation through the thermal sensitivity of the materials. In the scope of this study, the superelastic SMAs were employed with the aim of exploring their potential contributions to the seismic applications.

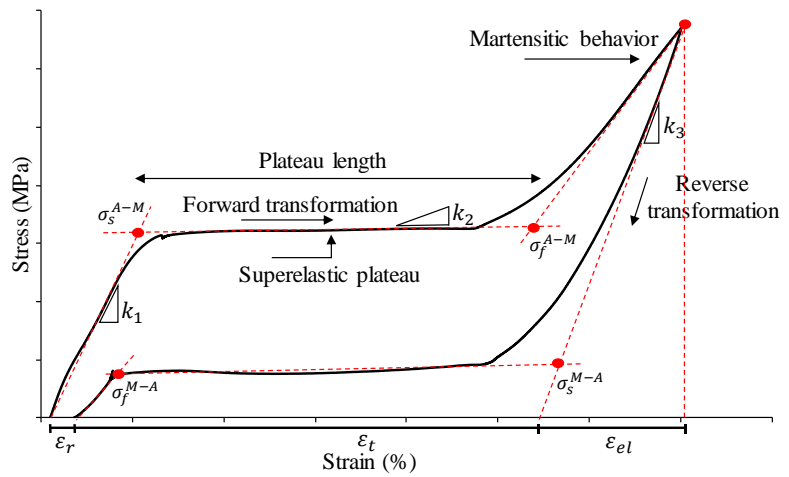

Fig. 1: Representative stress-strain relationship of superelastic SMAs.

Superelasticity is an isotherm process and phase transformation from martensite to austenite under an external load. Such a martensitic transformation of SMAs occurs only by stress excitation at a temperature above the austenitic finish $\left(\mathrm{A}_{\mathrm{f}}\right)$ temperature of the material. Initially, the austenitic alloy display an elastic behavior upon the application of stress level until the transformation stress $\left(\sigma_{S}^{A-M}\right)$. If the loading continues above this stress level, the SIM occurs following detwinning of the transformed martensite until the complete transformation at a strain level corresponding to $\sigma_{f}^{M-A}$. The upper plateau corresponds to the formation of the martensite while the lower plateau represents the reversion of the SIM when the stress is released. The entire recovery is completed in the form of flag-shaped stress-strain hysteretic loops through the forward and reverse transformation cycles. Due to defect generation mechanisms taking place during martensitic transformation, a residual strain, $\varepsilon_{r}$, can be induced in the material during deformation which will be carried over to the next stress cycle in case of incomplete recovery.

\section{Material Tests}

The Cu-Al-Mn and NiTi SMAs were acquired from Furukawa Techno Material Co. Ltd. (Japan) and Stanford Advanced Materials (U.S.A.), respectively. Then, tensile tests for $\mathrm{Cu}-\mathrm{Al}-\mathrm{Mn}$ and Ni-Ti SE SMAs as well as conventional steel were 
conducted on the dog-bone shaped coupon specimens, which were extracted from as-received Cu-Al-Mn, Ni-Ti SMA and Steel rods using wire electrical discharge machining. The tests were carried out using the INSTRON testing machine having $100 \mathrm{kN}$ force capacity in tension and compression. The details of the test setup are given in Fig. 2(a). The gauge dimensions for these specimens were around $12 \mathrm{~mm} \times 3 \mathrm{~mm} \times 1 \mathrm{~mm}$. The strain rate, $\dot{\varepsilon}$, and room temperature, $\mathrm{T}\left({ }^{\circ} \mathrm{C}\right)$, were $5 \times 10^{-4} \mathrm{~s}^{-1}$ and almost $24{ }^{\circ} \mathrm{C}$ during the tests, respectively. The strain rate used here is similar to that used in the study [6]. For the cyclic tests, a loading protocol starting with $0.5 \%$ and $1 \%$ strain values and continues until the fracture of the tested samples with a $1 \%$ strain increase at each cycle was adopted to the tests. The tested coupon specimens are shown in Fig. 2(b).

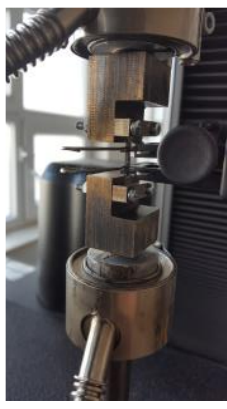

(a) Side view and back view

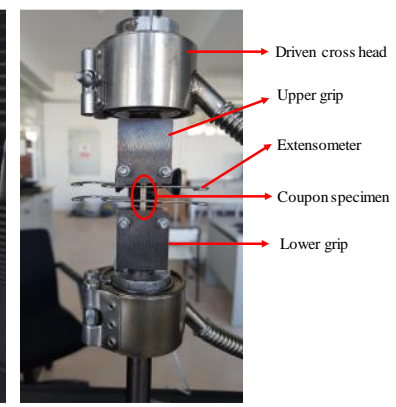

Fig. 2: View of the experimental setup and tested coupon specimens.
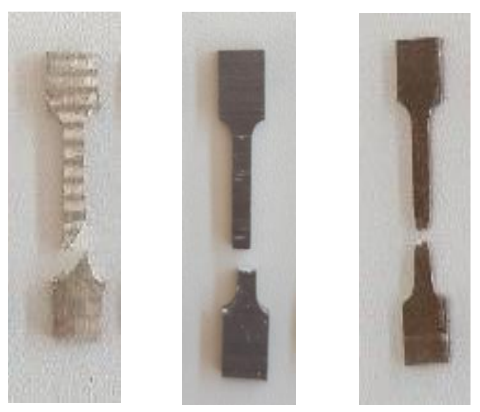

(b) $\mathrm{Cu}-\mathrm{Al}-\mathrm{Mn}, \mathrm{Ni}-\mathrm{Ti}$, and Steel (respectively)

\section{Material Test Results}

The graphical illustrations for the monotonic and cyclic tension test results are displayed in Fig. 3. For SE SMA specimens, the cyclic test results are divided into two parts; partial range and full range including the austenitic and martensitic behavior. In this presentation, partial range means stress cycling up to the strain level at which A-M transformation ends whereas the full range corresponds to stress cycling up to failure, including stress cycling of detwinned martensite for $\mathrm{Cu}-\mathrm{Al}-\mathrm{Mn}$ and Ni-Ti. Such a presentation can help to better understand the behavior of these materials under the given repeating axial loading conditions.

The cyclic tension test results are first examined to calculate the main mechanical parameters. Table 1 summarizes the results for the Steel whereas the results of the SE SMA material tests are given in Table 2. The characteristic values in Table 2 are computed from the first full transformation cycle, corresponding to a 7\% strain for $\mathrm{Cu}-\mathrm{Al}-\mathrm{Mn}$ and a $6 \%$ strain for NiTi. In Table 2, only martensite yield stresses are obtained from the monotonic tests. Apparently, each of the tested materials has a different nature when axially loaded. The conventional steel has the highest ultimate strain level with $24 \%$ while the $\mathrm{Cu}-\mathrm{Al}-\mathrm{Mn}$ and Ni-Ti SE SMAs have nearly $13.2 \%$ and $12.35 \%$ ultimate strain values, respectively. Nevertheless, the CuAl-Mn has the top ultimate strength with $975 \mathrm{MPa}$, which is almost $\% 9$ and 2.5 times larger than the Ni-Ti and Steel, respectively, while the ultimate strength of the $\mathrm{Ni}-\mathrm{Ti}$ is the highest one in monotonic tests. The yielding stress of the Ni-Ti is almost 2.25 times greater than the $\mathrm{Cu}-\mathrm{Al}-\mathrm{Mn}$ while the conventional steel yielded at around $332 \mathrm{MPa}$. It is clear that the modulus of elasticity of the Steel in the first ascending part is 3.7 and 3.1 times larger than the Cu-Al-Mn and Ni-Ti SMAs, respectively. There is, however, various nature in SMAs under tensile loading. They have two different elastic parts; in the first part they have the austenite modulus $\left(k_{1}\right)$, and in the second part, they have the martensite modulus $\left(k_{3}\right)$. In both parts, the austenite and martensite modulus values for Ni-Ti SMAs are larger than the $\mathrm{Cu}-\mathrm{Al}-\mathrm{Mn}$ ones. When it comes to evaluating the monotonic test results, the materials show different characteristics in stress and strain values in comparison to the cyclic test results. Early fracture of the Ni-Ti sample during cyclic deformation test might result from the damage accumulated during the low-cycle fatigue regime, i.e. each transformation cycle induces damage and reduces the durability of the material, such that sample fails at a smaller strain value compared to the monotonic tensile failure test, where the sample is loaded only once and up to failure. The possible reason why this phenomenon is not observed in Steel is the high ductility of the material, as can be seen in Fig. 2(b) from the severe plastic deformation in the area of fracture and necking. Similar fracture strain observed during cyclic deformation tests and monotonic failure tests of $\mathrm{Cu}-\mathrm{Al}-\mathrm{Mn}$ SMA needs further investigation and is beyond the scope of this paper. Fig. 3(f) indicates that the Ni-Ti has the highest yielding and ultimate stress as well as 
its large yielding strain with $0.57 \%$, corresponding to $\sigma_{S}^{A-M}$. By starting from $7 \%$ and $6 \%$ strain, the $\mathrm{Cu}-\mathrm{Al}-\mathrm{Mn}$ and $\mathrm{Ni}-\mathrm{Ti}$ are loaded beyond their elastic portion, respectively, and the martensitic transformations start. The superelastic plateau length of the $\mathrm{Cu}-\mathrm{Al}-\mathrm{Mn}$ is $6 \%$ while the $\mathrm{Ni}-\mathrm{Ti}$ has a $5 \%$ length in this parameter. The longer the plateau length, the more deformable the SE SMAs are under a similar load level.

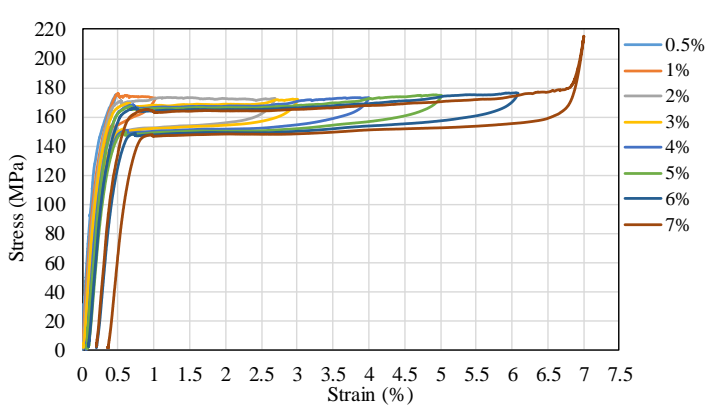

(a) Cyclic test of SE Cu-Al-Mn (Partial range)

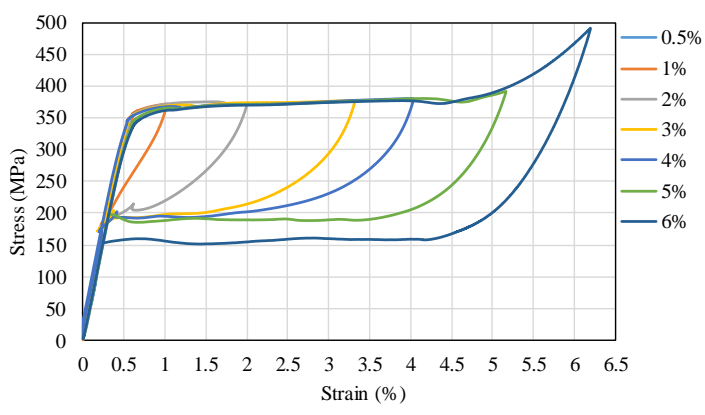

(c) Cyclic test of SE Ni-Ti (Partial range)

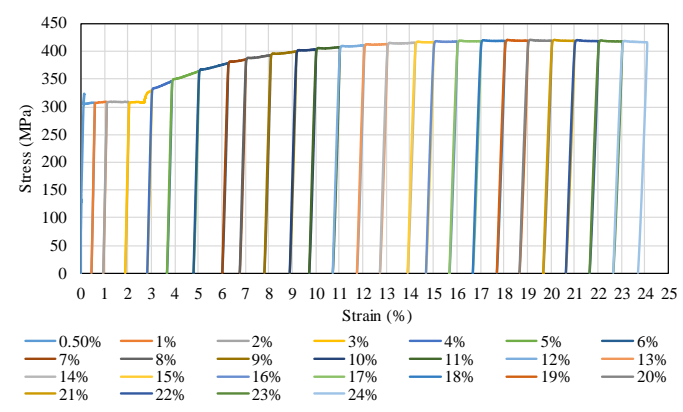

(e) Cyclic test of conventional steel

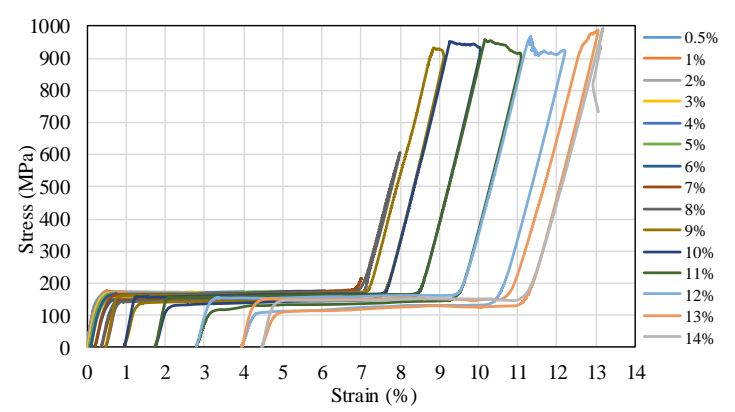

(b) Cyclic test of SE Cu-Al-Mn (Full range)

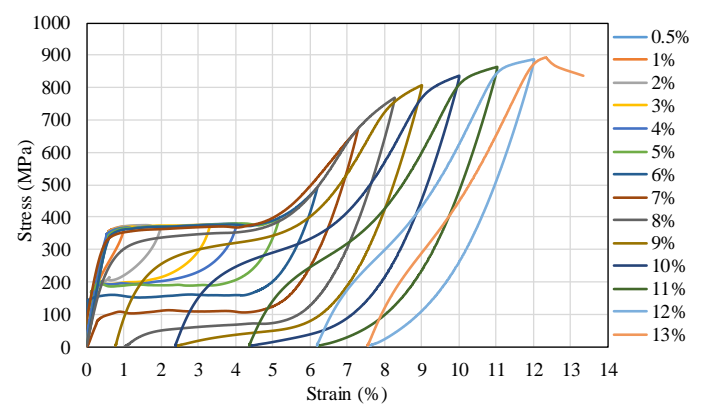

(d) Cyclic test of SE Ni-Ti (Full range)

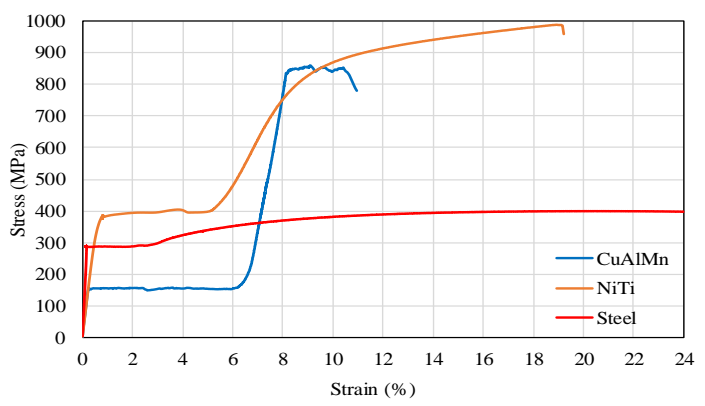

(f) Monotonic tensile test results

Fig. 3: Monotonic and cyclic tension test results of the materials.

Table 1: Measured mechanical properties of conventional steel.

\begin{tabular}{|c|c|}
\cline { 2 - 2 } \multicolumn{1}{c|}{} & Steel \\
\hline Yielding stress (MPa) & 332.5 \\
\hline Yielding strain (\%) & 0.165 \\
\hline Ultimate stress (MPa) & 435.2 \\
\hline Ultimate strain (\%) & 24 \\
\hline Young modulus (GPa) & 200 \\
\hline
\end{tabular}


Table 2: Measured mechanical properties of SE Cu-Al-Mn and Ni-Ti SMAs.

\begin{tabular}{|c|c|c|}
\cline { 2 - 3 } \multicolumn{1}{c|}{} & Cu-Al-Mn & Ni-Ti \\
\hline The austenite to martensite start stress, $\sigma_{s}^{A-M}(\mathrm{MPa})$ & 162 & 365 \\
\hline The austenite to martensite finish stress, $\sigma_{f}^{A-M}(\mathrm{MPa})$ & 174 & 385 \\
\hline The martensite to austenite start stress, $\sigma_{s}^{M-A}(\mathrm{MPa})$ & 156 & 165 \\
\hline The martensite to austenite finish stress, $\sigma_{f}^{M-A}(\mathrm{MPa})$ & 145 & 150 \\
\hline Martensite yield stress, $\sigma_{y}^{M}$ & 849 & 874.7 \\
\hline Residual strain, $\varepsilon_{r}$ & 0.16 & 0.2 \\
\hline Transformation strain, $\varepsilon_{t}$ & 6.22 & 5.16 \\
\hline Elastic strain, $\varepsilon_{e l}$ & 0.62 & 0.84 \\
\hline The austenite modulus, $k_{1}(\mathrm{MPa})$ & 54600 & 64500 \\
\hline The martensite modulus, $k_{3}(\mathrm{MPa})$ & 188 & 372 \\
\hline The transformation hardening coefficient, $k_{2}(\mathrm{MPa})$ & 34400 & 41200 \\
\hline The lower plateau stress factor, $\beta=1-\sigma_{f}^{M-A} / \sigma_{s}^{A-M}$ & 0.10 & 0.59 \\
\hline Ultimate strain, $\varepsilon_{u}(\%)$ & 13.2 & 12.35 \\
\hline Post yield stiffness ratio, $\alpha=k_{3} / k_{1}$ & 0.63 & 0.64 \\
\hline
\end{tabular}

\section{1. Residual Strain}

One of the key parameters to identify the effectiveness of SMAs is the residual strain upon stress recovery. While the residual strain, $\varepsilon_{r}$, is a sign of plastic deformation that occurs at loads above the yield stress for conventional engineering materials, it can take place in SMAs below the actual yield stress of martensite or austenite (Table 2) as a result of defect generation during repeated martensitic transformation. This defect generation occurs primarily to accommodate the transformation shear and volume change during transformation. $\varepsilon_{r}$ can be quantified as the difference between the loading and unloading points on the horizontal axis of the stress-strain curve, which is shown in Fig. 1. The cumulative residual strain values were calculated for each of the material whose test results are presented in Fig 3. It is evident from Fig. 4 that conventional steel is subjected to severe residual strains even in the beginning of loading. On the contrary, SE SMAs are exposed to relatively lower residual strains compared to that of Steel. Before the $\sigma_{f}^{M-A}$ stress level of the SMAs, the materials behaves almost elastically due to superelasticity. Then, comparatively small residual strain values begin to show up following the martensite-to-austenite transformation. At the end of the tests, the $\mathrm{Cu}-\mathrm{Al}-\mathrm{Mn}$ and Ni-Ti SMAs experienced nearly $4 \%$ and $8 \%$ residual strains, respectively. It shows the excellent performance of $\mathrm{Cu}-\mathrm{Al}-\mathrm{Mn}$ through its exceptional superelasticity.

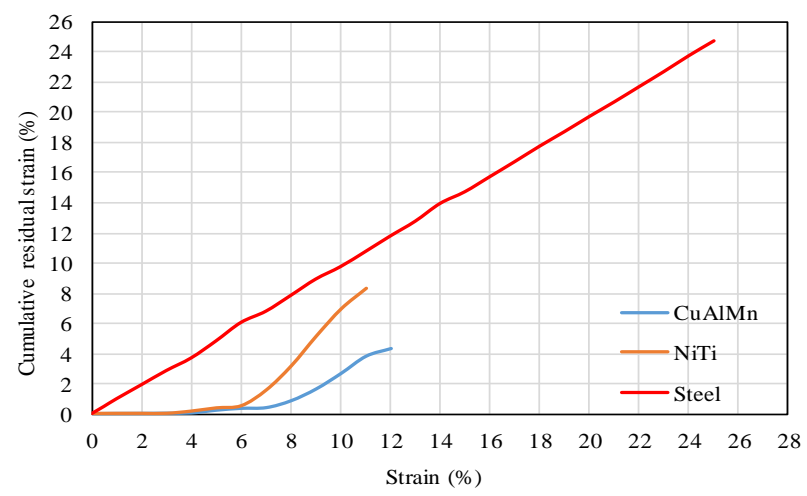

Fig. 4: Cumulative residual strain of the materials. 


\section{2. Strain recovery}

The second essential quantity regarding the tension test results is the strain recovery capacity of the materials. This parameter indicates the percentage of the strain recovered by the material upon the unloading. It is estimated from the ratio of the difference between the peak strain and unloading residual strain for each of cyclic tension loading (Eqn. 1).

$$
\frac{\varepsilon_{\max }-\varepsilon_{r}}{\varepsilon_{\max }} \times 100
$$

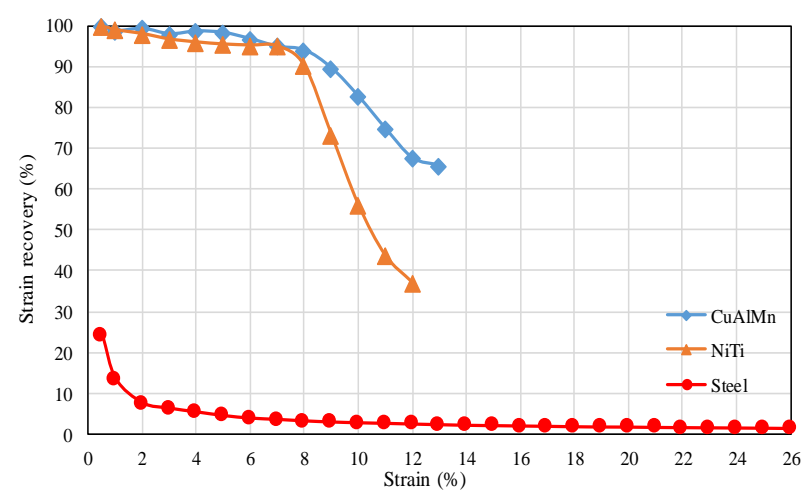

Fig. 5: Strain recovery capacity of the materials.

It is obvious from Fig. 5 that SMA materials exhibit superior performance in recovering the nonlinear deformations. Specifically, the strain recovery of the SMAs is over $90 \%$ up to $8 \%$ cyclic strain while the Steel recovers its plastic deformations in noticeably lower percentages. Furthermore, a decrease in the recovery capacity for SMAs begins after the martensitic deformations start and then visible but negligible residual deformations come out. Contrary to this, a significant reduction in the strain recovery can be seen in the early cyclic strains for conventional steel material. As a result, a good performance in recovery capacity, which is greater than $60 \%$, can be displayed in $\mathrm{Cu}-\mathrm{Al}-\mathrm{Mn}$ at the end of the tests.

\section{3. Energy dissipation}

Materials absorb and dissipate energy through their hysteretic behavior. When the energy dissipation of the materials is at issue, their stress-strain relationship is of importance. As seen in Fig. 3, the hysteretic characteristics of the materials differ from each other and this difference gives rise to a particular change in the amount of energy dissipation. Arising from the nature of the materials, a considerable amount of energy dissipation occurs during the loading-unloading process causing the expectation that pseudoelastic alloys could function as an effective damping material for incorporation in structures prone to seismic vibrations [7]. The energy dissipation capacity for each material was calculated from the area under the stress-strain curve enclosed within each strain cycle. Hence, the energy dissipation of the materials is given in Fig. 6.

Apparently, the Steel displays nearly linear behavior with average performance. Nevertheless, the Cu-Al-Mn shows the minimum performance in energy dissipation in the beginning whereas the amount of energy dissipation for Ni-Ti exceeds the Steel at around 4\% cyclic strain. As shown in Fig. 3, the enclosed area for Ni-Ti within each cyclic strain is visibly larger than the $\mathrm{Cu}-\mathrm{Al}-\mathrm{Mn}$. Variations in the enclosed areas cause a critical difference in the energy dissipation characteristics of the SMAs. An upward trend in the energy dissipation for Ni-Ti can be observed in the beginning of cyclic loadings due to its stress-strain nature with higher enclosed areas even before the $\sigma_{f}^{A-M}$ stress level. Additionally, at $12 \%$ strain level, the energy dissipated by the $\mathrm{Cu}-\mathrm{Al}-\mathrm{Mn}$ is almost three times lower than the Ni-Ti. At the end of the tests, the amount of energy dissipation for Ni-Ti is $40 \%$ larger than the conventional steel despite the comparatively higher ultimate strain of the Steel. 


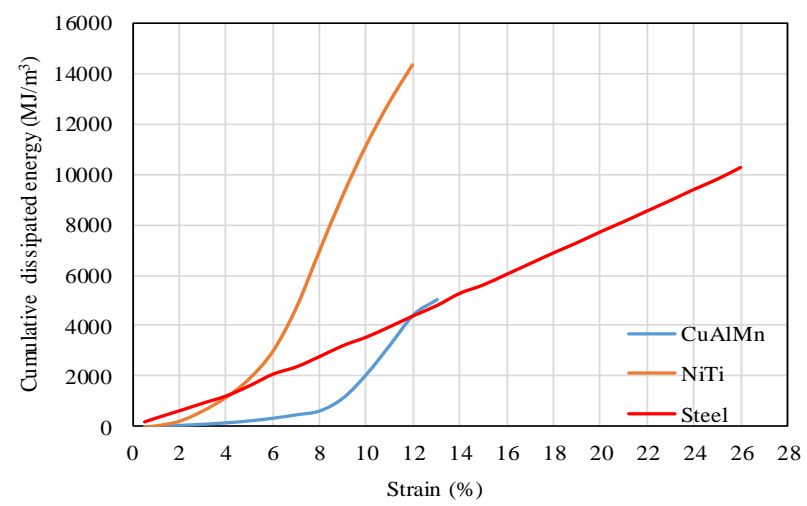

Fig. 6: Energy dissipation of the materials.

\section{4. Viscous damping}

Taking into consideration the hysteretic nature of the materials, they absorb the input energy causing the hysteretic response of the materials. In the superelastic form of the SMAs, they can show repeated hysteretic damping throughout the cyclic loading. The equivalent viscous damping parameter (EVD) is generally a key factor to measure the efficacy of a device in damping vibrations [8]. The potential of SMAs in energy dissipation and damping parameter is also valid in consecutive cyclic loading conditions [3]. Similar damping parameters were also calculated for SMA materials in [8, 9]. The equivalent viscous damping was computed using Eqn. 2, where $E_{D}$ is the dissipated energy during cyclic motion, $E_{s o}$ is the elastic strain energy, $A_{\text {loop }}$ is the enclosed area within the stress-strain curve of the imposed cycle, $\sigma_{\max }$ is the maximum stress and $\varepsilon_{\max }$ is the maximum strain in the loop under consideration.

$$
\xi_{\text {eq }}=\frac{1}{4 \pi} \frac{E_{D}}{E_{S o}}=\frac{1}{2 \pi} \frac{A_{\text {loop }}}{\sigma_{\max } \varepsilon_{\max }}
$$

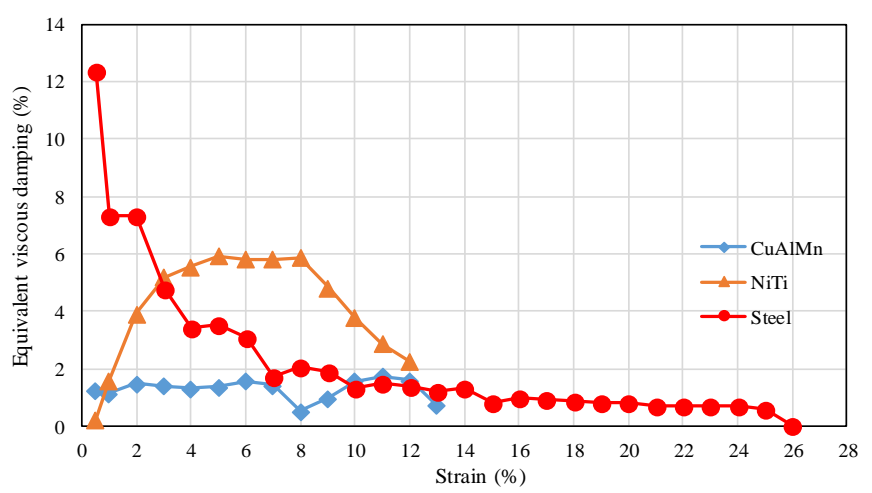

Fig. 7: Equivalent viscous damping of materials.

Fig. 7 illustrates the calculated EVD values for each of the tested materials. A sharp decline for Steel from $12.4 \%$ to $2 \%$ viscous damping at $8 \%$ strain level can be seen on the given graph and it continues to go down until the fracture point. However, SMAs demonstrate a stable performance during the loading. The $\mathrm{Cu}-\mathrm{Al}-\mathrm{Mn}$ has a steady path with an average EVD of $1.5 \%$ while the Ni-Ti exhibits a maximum of $6 \%$ equivalent viscous damping. A sudden decline in $\mathrm{Cu}-\mathrm{Al}-\mathrm{Mn}$ at the $8 \%$ cyclic strain, which is the second trend line for the martensitic phase, stems from considerably higher stress value with the similarly enclosed area in comparison to the previous $7 \%$ cyclic strain. The drop in the percentage of viscous damping for Ni-Ti after $8 \%$ strain level results from the decreasing dissipated energy while the stress level increases. 


\section{Conclusion}

In this study, superelastic Cu-Al-Mn and Ni-Ti dog-bone shaped SMA coupon specimens were subjected to monotonic and cyclic tensile tests in order to explore their potential for seismic mitigation in development of a new class of seismic resistance devices based on the SMAs, seismic design and other applications. Besides, conventional steel specimens were tested to compare the advantages and disadvantages of the SMAs. The following conclusions can be drawn based on the experimental test results:

- The SE SMAs clearly demonstrate superior performance in recovering plastic deformations compared to conventional steel. The Cu-Al-Mn specimens have the highest percentage in strain recovery with the lowest residual strain values. One of the main problems in recovering the structures after damaging natural disasters can be solved through the re-centering nature of these alloys. It could cause time-efficient solutions with minor damage conditions.

- The obvious increase in the amount of dissipated energy by the superelastic alloys starts after the onset of plastic deformations while the conventional steel shows linear and average performance in this parameter. The higher dissipation energy capacity of the superelastic alloys, specifically Ni-Ti, from the beginning of early strain values can make them favorably potential alloys in seismic mitigation. Moreover, the consecutive cyclic hysteresis of the superelastic alloys are critically important when considering earthquakes with cyclic motions.

- The Ni-Ti displays the best performance in equivalent viscous damping ratio with $6 \%$ whereas the $\mathrm{Cu}-\mathrm{Al}-\mathrm{Mn}$ has steady and lower damping values. Under repeated loading conditions, viscous damping is important to absorb the input energy resulting from ground motions based on the energy dissipation capacity of the materials. The test results indicate that the SE alloys can be used in seismic-resistant damping devices to absorb the input energy due to earthquake effects.

- The optimization can be done for SE SMAs to benefit from both austenitic and martensitic phases to obtain re-centering with higher energy dissipation and damping capacity.

\section{Acknowledgements}

This study was granted by Anadolu University with the project number of $1606 \mathrm{~F} 552$.

\section{References}

[1] B. Duran, "Seismic performance upgrading of reinforced concrete frames using shape memory alloys bars," M.Sc. Dissertation, Dept. Civil Eng., Anadolu University, Turkey.

[2] R. DesRoches and B. Smith, "Shape memory alloys in seismic resistant design and retrofit: a critical review of their potential and limitations," J. Earthq. Eng., vol. 8, no. 3, pp. 415-429, 2004.

[3] B. Duran, O. Tunaboyu, K. C. Atli, and Ö. Avşar, "Seismic performance upgrading of substandard RC frames using shape memory alloy bars," Smart Mater. Struct., vol. 28, no. 8, pp. 085007, 2019.

[4] Y. Araki, T. Endo, T. Omori, Y. Sutou, Y. Koetaka, R. Kainuma and K. Ishida, "Potential of superelastic Cu-Al-Mn alloy bars for seismic applications," Earthq. Eng. Struct. D., vol. 40, no. 1, pp. 107-115, 2011.

[5] N. A. Hamid, A. Ibrahim, A. Adnan and M. H. Ismail, "Pseudo Elastic and Self-healing Cyclic Properties of Smart Ni-Ti Shape Memory Alloy for Seismic Mitigation," in IOP Conference Series: Materials Science and Engineering, 2018, vol. 431, no. 7, pp. 072012, IOP Publishing.

[6] K. C. Atli, "The effect of tensile deformation on the damping capacity of Ni-Ti shape memory alloy," J. Alloy Comp., vol. 679, pp. 260-267, 2016.

[7] N. Shajil, D. Das and L. Chandrasekaran, "Effects of cycling on the pseudoelastic properties of Cu-Al-MnNi and TiNi based pseudoelastic alloys," Int. J. Struct. Chang. Solids, vol. 1, no. 1, pp. 171-185, 2009.

[8] M. Dolce, D. Cardone and R. Marnetto, "Implementation and testing of passive control devices based on shape memory alloys," Earthq. Eng Struct. D., vol. 29, no. 7, pp. 945-968, 2000.

[9] R. DesRoches, J. McCormick and M. Delemont, "Cyclic properties of superelastic shape memory alloy wires and bars," J. Struct. Eng., vol. 130, no. 1, pp. 38-46, 2000. 